\title{
Serial Estimation of Urinary Fibrin/ Fibrinogen Degradation Products in Kidney Transplantation
}

\author{
C. L. HALL, NOOSHABEH PEJHAN, R. W. THOMSON, P. DAWSON-EDWARDS, A. D. BARNES, \\ B. H. B. ROBINSON, M. J. MEYNELL, J. D. BLAINEY
}

transplant recipients has shown that acute rejection episodes are accompanied by and occasionally preceded by increased F.D.P. excretion which subsides with recovery from the rejection

\section{Summary}

The urinary excretion of fibrin/fibrinogen degradation products (F.D.P.) of 81 human cadaver kidney transplants has been measured serially by the techniques of tanned red cell haemagglutination inhibition immunoassay and immunonephelometry. Acute rejection episodes in functioning transplants have been associated with increased F.D.P. excretion which in $80 \%$ of cases has preceded clinical diagnosis by periods of one to seven days. Recovery from these episodes has been associated with a rapid fall of F.D.P. excretion to undetectable levels. The level of F.D.P. excretion during a rejection episode is a guide to its ultimate outcome. Irreversibly rejected kidneys excrete high levels of F.D.P. for long periods. Viable kidney transplants with prolonged oliguric phases can be distinguished, while still oliguric, from rejected kidneys by their low F.D.P. excretion. F.D.P. cannot usually be detected in the urine of well-functioning transplants. Episodes of raised F.D.P. excretion in the absence of acute clinical rejection, however, occur occasionally and may be associated with permanent impairment of renal function.

\section{Introduction}

Clinical criteria of kidney transplant rejection are unreliable and frequently inconclusive and it is often necessary to perform potentially hazardous procedures such as kidney biopsy and angiography. A simple test which could be carried out on the urine of a transplanted patient and would give a reliable indication of the condition of the transplant would be of great clinical value. Histological studies (Porter, 1967) have established that intrarenal fibrin deposition occurs during acute rejection episodes. Fibrin/fibrinogen degradation products (F.D.P.) have been shown by immunodiffusion and immunoelectrophoresis (Braun and Merrill, 1968; Antoine et al., 1969) in the urine of kidney transplant recipients, though the exact nature and source of these proteins remains uncertain. The use of the sensitive but laborious tanned red cell haemagglutination inhibition immunoassay (Clarkson et al., 1970) on the urine of kidney

\footnotetext{
Medical Research Council Renal Research Laboratory, Queen Elizabeth Hospital, Birmingham B15 2TH

C. L. HALL, M.B., M.R.C.P., Senior Registrar

J. D. BLAINEY, M.D., F.R.C.P., Consultant Physician

Artificial Kidney Unit, Queen Elizabeth Hospital, Birmingham B15 2TH

R. W. THOMSON, M.B., F.R.c.s., Senior Surgical Registrar

P. DAWSON-EDWARES, M.B., F.R.C.S., Consultant Surgeon

A. D. BARNES, M.CH., F.R.C.S., Consultant Surgeon

B. H. B. ROBINSON, M.D., M.R.C.P., Consultant Physician

Department of Haematology, Queen Elizabeth Hospital, Birmingham B15 2TH

NOOSHABEH PEJHAN, A.I.M.L.T., Senior Technician

M. J. MEYNELL, M.D., F.R.C.PATH., Consultant Haematologist
} episode. These findings have been confirmed using a much simpler latex slide agglutination technique (Hulme and Pitcher, 1973).

This paper reports the results of the serial estimation, by two different techniques, of the urinary F.D.P. excretion of a large number of kidney transplant patients in order to define its relation to transplant function and rejection and to assess its value in the clinical management of these patients.

\section{Patients}

Eighty-one cadaver kidney transplants were studied; 64 patients received a single transplant, 7 received a second, and 1 received a third transplant. Single early morning urine specimens were examined daily during the period in hospital and at each outpatient visit until stable renal function was achieved. Twentyeight patients with successful transplants were followed for periods of more than three months, including three for 12 months and one for 14 months. All urine samples were stored at $-20^{\circ} \mathrm{C}$ and were examined retrospectively without knowledge of the clinical status of the patient or his treatment. Prednisone and azathioprine in conventional dosage were given throughout. Rejection episodes were treated with prednisone $200 \mathrm{mg}$ daily for three days and actinomycin C $200 \mu \mathrm{g}$ daily for three days.

\section{Methods}

Tanned Red Cell Haemagglutination Inhibition Immunoassay (H.A.I.).-Unconcentrated transplant urine was used. To remove fibrinogen, thrombin was added to give a final concentration of 5 units $/ \mathrm{ml}$. The specimens were absorbed with concentrated sheep red cells and the F.D.P. content was determined by the technique of tanned red cell haemagglutination inhibition (Burroughs Wellcome). The maximum sensitivity was 1.25 $\mu \mathrm{g} / \mathrm{ml}$ (fibrinogen).

Immunonephelometry (I.N.).-The method of Farrell and Wolf (1972) was used. To remove fibrinogen, thrombin was added to unconcentrated transplant urine to give a final concentration of 5 units $/ \mathrm{ml}$. After centrifugation the supernatant was removed and $1 / 30$ dilution made in a hypertonic buffer $(17 \mathrm{~g} \mathrm{NaCl} / 100 \mathrm{ml}$ and $1.5 \mathrm{~g}$ dipotassium edetate $/ 100 \mathrm{ml}$ ); $0.2 \mathrm{ml}$ of sheep antihuman fibrinogen antiserum was added. After 30 minutes the estimation was carried out by means of a Thorp micronephelometer against standards prepared from human plasma of known fibrinogen content. The maximum sensitivity of the technique was $1.5 \mu \mathrm{g} / \mathrm{ml}$ (fibrinogen).

\section{Results}

Successful Transplants.-In 39 patients the kidney transplants functioned without any evidence of rejection. A characteristic pattern of urinary F.D.P. excretion was observed in these cases (fig. 1). High levels were present in the oliguric phase. A rapid fall to low levels occurred at or before the onset of the diuretic 

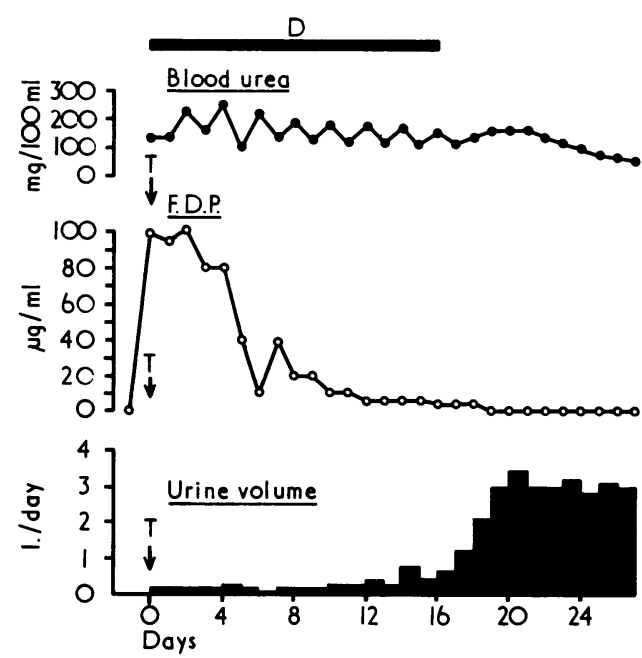

FIG. 1-Urinary F.D.P. excretion in patient with successful kidney transplant without rejection episodes. $\mathbf{T}=$ Transplant operation.

phase and thereafter F.D.P. could not be detected in the urine of the well-functioning transplant. This pattern of F.D.P. excretion was similar to that observed in non-transplanted patients recovering from acute tubular necrosis of their own kidneys.

Acute Rejection Episodes.-There were 30 acute rejection episodes diagnosed clinically according to the following criteria: (1) tender, swollen kidney transplant, (2) pyrexia, (3) decreasing urinary output, (4) rising blood urea and serum creatinine, (5) weight gain, (6) rising blood pressure. Nineteen rejection episodes occurred after the onset of the diuretic phase. In two cases urine specimens were not collected at the appropriate time. The remaining 17 showed increased F.D.P. excretion at the time of the rejection episodes. In 14 of the 17 the increased F.D.P. excretion preceded the clinical diagnosis of rejection by periods of one to seven days and in the remaining three cases occurred at the time of clinical diagnosis (table I). Of the 19 kidneys 15 survived

TABLE I-Timing of Increased F.D.P. Excretion versus Clinical Diagnosis in Rejection Episodes

\begin{tabular}{c|c|c|c}
\hline Patient & $\begin{array}{c}\text { Day of Increased } \\
\text { F.D.P. Excretion }\end{array}$ & $\begin{array}{c}\text { Day of Clinical } \\
\text { Diagnosis of } \\
\text { Rejection }\end{array}$ & $\begin{array}{c}\text { No. of Days F.D.P. } \\
\text { Excretion was In- } \\
\text { creased before } \\
\text { Clinical Diagnosis } \\
\text { of Reiection }\end{array}$ \\
\hline 1 & 18 & 18 & 0 \\
2 & 13 & 16 & 3 \\
3 & 14 & 19 & 5 \\
4 & 4 & 5 & 1 \\
5 & 42 & 43 & 7 \\
6 & 15 & 22 & 3 \\
7 & 29 & 32 & 4 \\
8 & 34 & 52 & 1 \\
9 & 21 & 22 & 1 \\
10 & 55 & 56 & 2 \\
12 & 45 & 29 & 0 \\
13 & 27 & 6 & 2 \\
14 & 6 & 23 & 3 \\
15 & 21 & 106 & 2 \\
16 & 103 & & \\
17 & 21 & &
\end{tabular}

the rejection episodes, the urinary F.D.P. excretion falling to unrecordable levels as kidney function recovered (fig. 2). Four kidneys were rejected and high F.D.P. excretion continued until nephrectomy was performed (fig. 3). The mean maximum F.D.P. excretion of the kidneys destroyed by the rejection episodes was four to nine times greater than that of the kidneys which recovered (table II). Eleven rejection episodes occurred during the oliguric phase, at which time F.D.P. excretion is normally at a high level, and in only three cases was a further increase in urinary F.D.P. observed. Five of these transplants survived the rejection episodes, the urinary F.D.P. subsequently falling to unrecordable levels at the onset of the diuretic phase. Six were destroyed and high F.D.P. levels persisted until nephrectomy was carried out.

TABLE II-Maximum F.D.P. Excretion during Rejection Episode. Results are given in $\mu \mathrm{g} / \mathrm{ml}$

\begin{tabular}{|c|c|c|}
\hline Patient & H.A.I. & I.N. \\
\hline $\begin{array}{l}\mathbf{A} \\
\mathbf{B} \\
\mathbf{C} \\
\mathbf{D} \\
\mathbf{E} \\
\mathbf{F} \\
\mathbf{G} \\
\mathbf{H} \\
\mathbf{I} \\
\mathbf{J} \\
\mathbf{K}\end{array}$ & $\begin{array}{r}\text { Rejection evith Survivo } \\
160 \\
640 \\
40 \\
20 \\
20 \\
40 \\
40 \\
40 \\
40\end{array}$ & $\begin{array}{l}20 \\
37 \\
50 \\
50 \\
50 \\
40 \\
57\end{array}$ \\
\hline Mean & 103 & 43 \\
\hline \multicolumn{3}{|c|}{ Rejection with Destruction of Transplant } \\
\hline $\begin{array}{l}\mathbf{L} \\
\mathbf{M} \\
\mathbf{N} \\
\mathbf{O}\end{array}$ & $\begin{array}{l}320 \\
320 \\
640\end{array}$ & $\begin{array}{l}375 \\
400\end{array}$ \\
\hline Mean & 430 & $387 \cdot 5$ \\
\hline
\end{tabular}
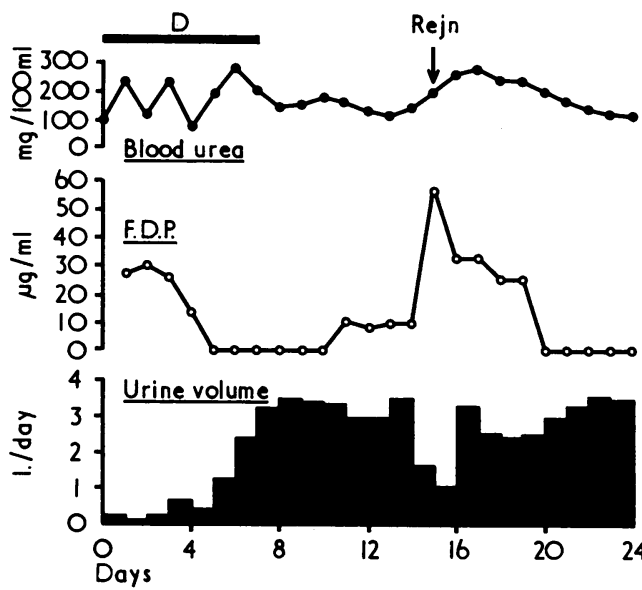

FIG. 2-Patient 2, male. Urinary F.D.P. excretion during early acute rejection episode which was treated successfully. Transplant operation performed on day 0 .

Non-functioning Transplants.-In 14 patients the kidney transplants failed to function and were removed. Rejection episodes were not diagnosed clinically in any of these patients. In three cases hyperacute rejection was shown histologically by a kidney biopsy taken at the completion of the ureteric anastomosis during the transplant operation. In five patients thrombosis of the renal artery at the site of the anastomosis was responsible and in six percutaneous kidney biopsy at a later date showed changes of advanced rejection. In all of these cases high urinary F.D.P. excretion persisted until nephrectomy was carried out.

Transplants with Prolonged Oliguric Phases (table III).--In 11 patients the transplants had oliguric phases of longer than 20 days but eventually functioned. In 10 of these patients the F.D.P. excretion was $10 \mu \mathrm{g} / \mathrm{ml}$ or less from the 20th day onwards. The F.D.P. excretion of the remaining patient (patient 28, table III), who suffered an early acute rejection episode which the transplant survived, fell to $10 \mu \mathrm{g} / \mathrm{ml}$ by the 25 th day and zero by the 30th. In contrast, the F.D.P. excretion of 8 of the 10 patients with rejected transplants was more than $20 \mu \mathrm{g} / \mathrm{ml}$ on the 20th day and continued at a high level thereafter. The two 


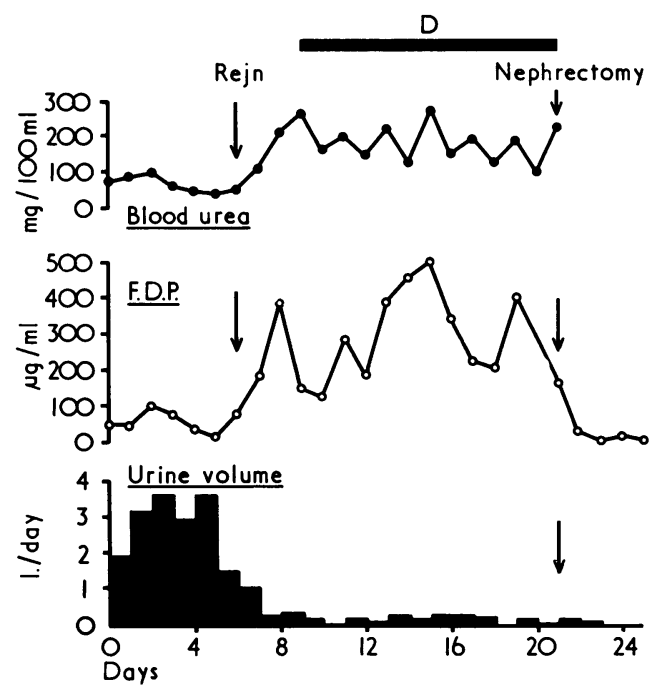
FIG. 3-Patient 14, male. Urinary F.D.P. excretion during acute rejection tion on day 0 . (Note: Persistently high F.D.P. levels from onset of rejection episode until transplant nephrectomy.)

TABLE III-Urinary F.D.P. Excretion 15-30 Days after Transplantation. Excretion is given in $\mu \mathrm{g} / \mathrm{ml}$

\begin{tabular}{|c|c|c|c|c|c|}
\hline Patient & Day 15 & Day 20 & Day 25 & Day 30 & $\begin{array}{c}\text { Onset of } \\
\text { Diuresis } \\
\text { (Day) }\end{array}$ \\
\hline \multicolumn{6}{|c|}{ Successful Transplants with Oliguric Phases Longer than 20 Days } \\
\hline $\begin{array}{l}18 \\
19 \\
20 \\
21 \\
22 \\
23 \\
24 \\
25 \\
26 \\
27 \\
28 *\end{array}$ & $\begin{array}{r}22 \\
10 \\
40 \\
20 \\
10 \\
20 \\
20 \\
5 \\
5 \\
5 \\
160\end{array}$ & $\begin{array}{c}8 \\
2 \\
10 \\
10 \\
5 \\
10 \\
5 \\
2 \cdot 5 \\
0 \\
0 \\
30\end{array}$ & $\begin{array}{r}2 \\
0 \\
5 \\
2 \\
0 \\
5 \\
0 \\
0 \\
0 \\
10\end{array}$ & $\begin{array}{l}0 \\
0 \\
5 \\
0 \\
2 \cdot 5 \\
2 \cdot 5 \\
0 \\
0 \\
0 \\
0\end{array}$ & $\begin{array}{l}26 \\
29 \\
44 \\
36 \\
23 \\
24 \\
27 \\
22 \\
21 \\
28 \\
23\end{array}$ \\
\hline Mean & 29 & $7 \cdot 5$ & $2 \cdot 4$ & 1 & \\
\hline Patient & Day 15 & Day 20 & Day 25 & Day 30 & $\begin{array}{c}\text { Nephrectomy } \\
\text { (Day) }\end{array}$ \\
\hline \multicolumn{6}{|c|}{ Rejected Transplants. Nephrectomy } \\
\hline $\begin{array}{l}29 \dagger \\
30 \\
31 \\
32 \ddagger \\
33 \\
34 \\
35 \\
365 \\
37 \\
38\end{array}$ & $\begin{array}{r}10 \\
320 \\
160 \\
5 \\
320 \\
320 \\
80 \\
40 \\
120\end{array}$ & $\begin{array}{r}5 \\
320 \\
40 \\
5 \\
80 \\
320 \\
420 \\
40 \\
90 \\
160\end{array}$ & $\begin{array}{r}320 \\
35 \\
5 \\
160 \\
640 \\
640 \\
20 \\
160\end{array}$ & $\begin{array}{r}320 \\
20 \\
160 \\
640 \\
640 \\
20\end{array}$ & $\begin{array}{l}36 \\
22 \\
36 \\
29 \\
34 \\
60 \\
33 \\
31 \\
24 \\
26\end{array}$ \\
\hline Mean & 153 & 148 & $247 \cdot 5$ & 300 & \\
\hline
\end{tabular}

* Acute rejection on day 1 with subsequent recovery.

$\dagger$ Acute rejection day 23 causing destruction of transplant.

₹ Patient secreting 31 . urine daily through own kidneys.
S Patient secreting 21 . urine daily through own kidneys.

patients with rejected transplants whose F.D.P. excretion was less than $10 \mu \mathrm{g} / \mathrm{ml}$ on day 20 were patient 29 , whose viable transplant was destroyed on day 23 by an acute rejection, and patient 32, who was secreting 31 . of urine daily through his own kidneys and hence diluting the F.D.P. excretion.

Long-term Results. - The urinary F.D.P. excretion of 28 patients with successful transplants was measured at each outpatient visit for periods of up to 14 months. In six patients F.D.P. were not detected in the urine at any time during the follow-up period. The remaining 22 patients showed 28 episodes of raised F.D.P. excretion (more than $10 \mu \mathrm{g} / \mathrm{ml}$ ). In none of these episodes was rejection diagnosed clinically. In six, however, mild but permanent impairment of renal function was observed. Increased F.D.P. excretion has been observed in many cases of glomerulonephritis (Clarkson et al., 1971); however, none of these 28 patients had any evidence of recurrent nephritis in their kidney transplants. Thus these episodes of raised F.D.P. excretion cannot be explained on this basis.

\section{Discussion}

A good correlation was observed between the results of the two techniques used in this study. The immunonephelometric technique has the advantage of being inexpensive, rapid, quantitative, and very sensitive, detecting fibrinogen in concentrations of $1-2 \mu \mathrm{g} / \mathrm{ml}$. Employing these techniques we have confirmed the reports of Clarkson et al. (1970) and Hulme and Pitcher (1973) that episodes of acute rejection in functioning kidney transplants are associated with increased F.D.P. excretion, which in $80 \%$ of cases preceded clinical diagnosis by periods of one to seven days. Increased urinary F.D.P. excretion has not been associated with acute urinary tract infection or perirenal haemorrhage. Thus the F.D.P. excretion is of value in confirming a clinical diagnosis of rejection and distinguishing it from other complications. In addition it may allow prediction that a rejection episode is imminent and permit increased immunosuppression at an early stage before a severe rejection is established with its attendant renal damage or destruction.

The level of the F.D.P. excretion correlates well with the severity of the rejection process. Kidneys destroyed in rejection episodes have excreted levels of $300-400 \mu \mathrm{g} / \mathrm{ml}$ compared with the $40-100 \mu \mathrm{g} / \mathrm{ml}$ of those which have survived. Thus an assessment can be made of the severity and prognosis of a rejection episode. A rising level of F.D.P. is a bad prognostic sign and an indication that further immunosuppression is required. Only one out of five severe rejection episodes, however (F.D.P. $300-600 \mu \mathrm{g} / \mathrm{ml}$ ), has been reversed successfully. The recovery from a rejection episode is associated with a fall in F.D.P. excretion to undetectable levels. Irreversibly rejected kidneys, the result of either an acute rejection episode or a clinically silent rejection process, continue to excrete significant levels of F.D.P. (more than $20 \mu \mathrm{g} / \mathrm{ml}$ ) for long periods. This enables these rejected kidneys to be distinguished from viable transplants with prolonged oliguric phases. The viable kidneys by the 20 th day after transplantation, though oliguric, excrete only low levels $(10 \mu \mathrm{g} / \mathrm{ml}$ or less) of F.D.P. in the urine. A good example of this was a patient whose F.D.P. excretion was less than $10 \mu \mathrm{g} / \mathrm{ml}$ by the 20 th day but whose transplant did not function until the 44th.

The long-term study of patients with functioning transplants has confirmed the findings of Clarkson et al. (1970) that episodic increases of F.D.P. excretion in the absence of clinical rejection episodes are not unommon. In $20 \%$ of these episodes, however, mild but permanent impairment of renal function has been observed. Whether increased immunosuppression at these times will prevent or delay renal damage remains to be determined.

The value of the urinary F.D.P. excretion in the first 10 days after transplantation, with the exception of transplants with immediate function, is limited by the high levels associated with the recovering acute tubular necrosis in the cadaver transplant. However, from the 15th day onwards it becomes a valuable measurement in the assessment of human kidney transplants. Most acute rejection episodes occur within three months of transplantation and during this period frequent testing is performed. Clearly large numbers of specimens have to be examined and such a laboratory burden can be accommodated conveniently only by an automated technique. Of the techniques used to measure urinary F.D.P. the immunonephelometric is the only one which lends itself to automation. This possibility is under investigation at the present time.

Requests for reprints should be addressed to: Dr. C. L. Hall, Medical Research Council Renal Research Laboratory, Clinical Research Block, Queen Elizabeth Hospital, Birmingham B15 2TH. 


\section{References}

Antoine, B., Neveu, T., and Ward, P. D. (1969). Transplantation, 8, 98. Braun, W. E., and Merrill, J. P. (1968). New England fournal of Medicine, 278, 1366 .

Clarkson, A. R., Morton, J. B., and Cash, J. D. (1970). Lancet, 2, 122.
Clarkson, A. R., MacDonald, M. K., Petrie, J. J. B., Cash, J. D., and Robson, J. S. (1971). British Medical fournal, 3, 447.

Farrell, G. W., and Wolf, P. (1972). Fournal of Immunological Methods, 1, 217 Hulme, B., and Pitcher, P. M. (1973). Lancet, 2, 6.

Porter, K. A. (1967). Fournal of Clinical Pathology, 20, Suppl., p. 518.

\title{
Androsterone/Etiocholanolone Ratios in Male Homosexuals
}

\author{
M. SYDNEY MARGOLESE, OSCAR JANIGER
}

British Medical fournal, 1973, 3, 207-210

\section{Summary}

Analyses of 24-hour specimens of urine from healthy adult males for androsterone and etiocholanolone produced values which, when calculated as discriminant scores, discriminated between heterosexual and exclusively homosexual individuals. This confirms a previous study.

No significant differences were found between heterosexuals and homosexuals in parental ages, secondary sex characteristics, genitalia, anthropometry, 17-ketosteroids, and 17-ketogenic steroids.

A significant difference was found between the heterosexual group and homosexual group in the number of homosexual relatives in the immediate and extended families.

\section{Introduction}

In the past few years the concept of a biological basis for homosexual behaviour has been reconsidered as a consequence of more precise investigative techniques. A previous study by Margolese (1970) showed that healthy adult males excreted androsterone and etiocholanolone in ratios that clearly discriminated between heterosexual and homosexual subjects. This was followed by a study by Lorraine (1970) who found low urinary testosterone in two homosexual males and raised urinary testosterone in four homo sexual females. The idea that changes in endocrine function might contribute to the genesis of homosexuality in humans was then given further impetus by Kolodny et al. (1971) who showed that plasma testosterone levels of a homosexual group were significantly lower than those of a control group. When investigating several biochemical parameters Evans (1972) found that homosexuals had a lower androsterone/ etiocholanolone (A/E) ratio, and higher 11-ketoetiocholanolone than heterosexuals.

Testosterone, the major natural androgen, is largely secreted by the testis as a result of stimulation by the luteinizing hormone from the anterior pituitary. The adrenal cortex secretes a small amount of testosterone and dehydroepiandrosterone (DHA), which may also be a precursor of testosterone. The intermediate metabolism of testosterone

\footnotetext{
School of Medicine, University of California Medical Center, Los Angeles, California, U.S.A.

M. SYDNEY MARGOLESE, M.D., Assistant Clinical Professor of Medicine (at present: Teacher, University of California Extension)

Department of Psychiatry and Human Behaviour, California College of Medicine, University of California, Irvine, and the Metropolitan State Hospital, Norwalk, California, U.S.A.

OSCAR JANIGER, M.D., Lecturer
}

has not been fully clarified. The liver converts testosterone by reduction to androsterone and etiocholanolone, which are stereo isomers, and they are conjugated mainly as glucuronides, and excreted in the urine. These two compounds plus DHA are the principal compounds making up the urinary 17-ketosteroids (17-KS), and are included in the analysis of urinary 17-KS. Of the total 17-KS about twothirds come from the adrenal cortex chiefly as DHA. When tested by biological assay almost all of the androgenic activity is found to be due to androsterone. The average normal adult $17-\mathrm{KS}$ excretion is $14 \mathrm{mg} / 24 \mathrm{hr}$ for men with a range of $9-22 \mathrm{mg}$ by the assay method used.

The present study was undentaken to investigate a larger number and greater variety of subjects than previously, and to provide a more detailed psychological evaluation.

\section{Subjects and Methods}

A total of 63 male subjects were studied, which included two sets of identical twins. The heterosexual controls were obtained from college student populations. The homosexual subjects were obtained from four homosexual organizations (see acknowledgements).

One of us (M.S.M.) was responsible for the physical evaluation and endocrine studies. This consisted of a brief metabolic history, determination of blood pressure and pulse, and notations of sexual hair distribution, genitalia, and anthropometric measurements. A blood sample was drawn for determination of the SMA 12 blood chemistry profile and free thyroxine. Each subject submitted a 24-hour specimen of urine which was analyzed for 17-ketosteroids, androsterone, and etiocholanolone. In the case of six homosexuals the urines were also analyzed for 17-ketogenic steroids. All blood and urine determinations were done by Bio-Science Laboratories of Van Nuys, California. The 17-ketosteroids were determined by a modification (Sobel et al., 1958) of the method of Drekter et al. (1952). Androsterone and etiocholanolone were determined by the method of Brooks (1958).

In the previous study a statistical analysis was done on the androsterone and etiocholanolone values by linear discriminant analysis. This provided a factor for androsterone $(0.231)$ and for etiocholanolone $(0.190)$ and their difference $(0.231 \mathrm{~A}-0.190 \mathrm{E})$ gave a discriminant score. These factors are being used in the present study as a test of their ability to discriminate between two new populations.

Each subject was interviewed by one of us (O.J.) for one and a half to two hours, using a modified Kinsey sexual inventory. The Kinsey heterosexual-homosexual rating scale (Kinsey et al., 1948) was used for classifications, as follows: 0 exclusively heterosexual; 1 predominately heterosexual, only incidentally homosexual; 2 predominately heterosexual, but more than incidentally homosexual; 3 equally heterosexual and homosexual; 4 predominately homosexual, but more than incidentally heterosexual; 5 predominately homo- 